\title{
PERFORMANCE OF RICHARDSON EXTRAPOLATION ON SOME NUMERICAL METHODS FOR A SINGULARLY PERTURBED TURNING POINT PROBLEM WHOSE SOLUTION HAS BOUNDARY LAYERS
}

\author{
Justin B. Munyakazi and Kailash C. Patidar
}

\begin{abstract}
Investigation of the numerical solution of singularly perturbed turning point problems dates back to late 1970s. However, due to the presence of layers, not many high order schemes could be developed to solve such problems. On the other hand, one could think of applying the convergence acceleration technique to improve the performance of existing numerical methods. However, that itself posed some challenges. To this end, we design and analyze a novel fitted operator finite difference method (FOFDM) to solve this type of problems. Then we develop a fitted mesh finite difference method (FMFDM). Our detailed convergence analysis shows that this FMFDM is robust with respect to the singular perturbation parameter. Then we investigate the effect of Richardson extrapolation on both of these methods. We observe that, the accuracy is improved in both cases whereas the rate of convergence depends on the particular scheme being used.
\end{abstract}

\section{Introduction}

We consider the problem

$$
\begin{gathered}
L u:=\varepsilon u^{\prime \prime}+a(x) u^{\prime}-b(x) u=f(x), x \in \Omega=(-1,1), \\
u(-1)=A, u(1)=B,
\end{gathered}
$$

where $A$ and $B$ are given real constants and $\varepsilon \in(0,1]$, and the coefficients $a(x), b(x)$ and $f(x)$ are sufficiently smooth functions in $\bar{\Omega}$.

The distinct zeros $\alpha_{i}, i=1,2, \ldots, r$ of $a(x)$ in the interval $\bar{\Omega}$, if they exist, are called the turning points of (1.1)-(1.2), provided that $a(-1) a(1) \neq 0$.

Received May 20, 2013; Revised February 3, 2014.

2010 Mathematics Subject Classification. 34E20, 65L10, 65L12, 65L70, 65L99.

Key words and phrases. singular perturbations, turning point problems, boundary layers, fitted operator finite difference methods, fitted mesh finite difference method, Richardson extrapolation, error estimates.

The second author was supported by the South African National Research Foundation. 
Berger et al. [7] showed that the bounds of the solution $u(x)$ near a given turning point $\alpha_{i}$ depend on $\varepsilon$ and the constants $\beta_{i}=b\left(\alpha_{i}\right) / a^{\prime}\left(\alpha_{i}\right)$. For $\beta_{i}<0$, $u(x)$ is "smooth" near $x=\alpha_{i}$ whereas $\beta_{i}>0$ indicates that $u(x)$ has a large gradient at $x=\alpha_{i}$ resulting in an "interior layer". On the other hand, $u(x)$ has a boundary layer at $x=-1$ and $x=1$ if and only if $a(-1)>0$ and $a(1)<0$, respectively.

In the rest of this paper, we assume that

$$
a(0)=0, a^{\prime}(0) \leq 0, a(-1)>0, \text { and } a(1)<0,
$$

thus ensuring that the classical solution to (1.1)-(1.2) has two exponential boundary layers. Also it is required that $b(x) \geq b_{0}>0$ so as to ensure that the solution of (1.1)-(1.2) satisfies a minimum principle. The condition $\left|a^{\prime}(x)\right| \geq\left|a^{\prime}(0) / 2\right|,-1 \leq x \leq 1$ guarantees the uniqueness of the turning point in the interval $[-1,1]$. Furthermore, we assume that $|a(x)-x b(x)| \geq \eta>0$ to ensure the stability of the solution.

Under the above assumptions, the operator $L$ admits the following continuous minimum principle.

Lemma 1.1. Let $\xi$ be a smooth function satisfying $\xi(-1) \geq 0, \xi(1) \geq 0$ and $L \xi(x) \leq 0, \forall x \in(-1,1)$. Then $\xi(x) \geq 0, \forall x \in[-1,1]$.

Proof. Let $x^{*} \in[-1,1]$ such that $\xi\left(x^{*}\right)=\min _{x \in[-1,1]} \xi(x)$ and assume $\xi\left(x^{*}\right)<$ 0 . Then, obviously, $x^{*} \notin\{-1,1\}, \xi^{\prime}\left(x^{*}\right)=0$ and $\xi^{\prime \prime}\left(x^{*}\right) \geq 0$. We have

$$
L \xi\left(x^{*}\right)=\varepsilon \xi^{\prime \prime}\left(x^{*}\right)+a\left(x^{*}\right) \xi^{\prime}\left(x^{*}\right)-b\left(x^{*}\right) \xi\left(x^{*}\right)>0,
$$

which is a contradiction. It follows that, $\xi\left(x^{*}\right) \geq 0$ and thus, $\xi(x) \geq 0, \forall x \in$ $[-1,1]$.

The minimum principle implies the uniqueness and existence of the solution. (as for linear problems, the existence of the solution is implied by its uniqueness.) We use this principle to prove the following results which states that the solution depends continuously on the data.

Lemma 1.2. Let $u(x)$ be the solution of (1.1)-(1.2). Then, we have

$$
\|u\| \leq C\left(b_{0}^{-1}\|f\|+\max \{|A|,|B|\}\right), \forall x \in[-1,1],
$$

where $\|\cdot\|$ denotes the maximum norm.

Proof. Consider the comparison function

$$
\Pi^{ \pm}(x)=b_{0}^{-1}|| f||+\max \{|A|,|B|\} \pm u(x) .
$$

Then we have

$$
L \Pi^{ \pm}(x)= \pm f(x)-\frac{b(x)}{b_{0}}\|f\|-b(x) \max \{|A|,|B|\} \leq 0 .
$$

implying that $\Pi^{ \pm}(x) \geq 0, \forall x \in[-1,1]$, which completes the proof. 
Numerous works have been devoted to studying singularly perturbed turning point problems (SPTPPs) of which we give some examples below.

Abrahamsson [1] and Berger et al. [7] derived a number of a priori estimates for solutions of SPTPPs. Adžić in [2], [3] and [5] developed modified standard spectral methods for singularly perturbed problems without turning points, with turning point and boundary layers and with turning point and interior layer, respectively. The same author used a domain decomposition method (in [4]) to solve some turning point problems via the asymptotic behavior of the exact solution. Patidar [22, 23] designed a fitted operator finite difference method (FOFDM) and a fitted mesh finite difference method (FMFDM), both along with the Numerov's method to solve a singularly perturbed self-adjoint problem. Some other works were considered in $[11,10]$ for various classes of singular perturbation problems. However, as far as the fitted finite difference methods are concerned, very little work is seen for the SPTPPs. Readers may wish to refer to $[15,16,25,28]$ for some additional readings on TPPs.

Recently, we designed a FOFDM to solve problem (1.1)-(1.2) [20]. In this paper, we propose a FMFDM to solve the problem above. Then we will study the effect of Richardson extrapolation on these two methods in order to determine whether this procedure improves the accuracy and/or the rate of convergence of these methods.

Richardson extrapolation is a procedure through which numerical approximations of a particular quantity are computed on different but nested meshes. A linear combination of these approximations yields a better accuracy as compared to when each of them taken separately. It is also expected that this procedure will improve the rates of convergence of these numerical approximations [27]. In the past, an almost first order convergence is improved to an almost second order convergence in [21] via extrapolation based on fitted mesh finite difference method for a convection diffusion problem. Likewise, we studied in [18], the convergence acceleration properties of extrapolation based on two different FOFDMs to solve a self-adjoint problem. We observed some improvement in one case. Also, we investigated the effects of this technique on a FMFDM to solve a self-adjoint problem in [19] and no improvement were observed. Note that, in all the cases, Richardson extrapolation improves the accuracy of the underlying method. For more details about Richardson extrapolation, the interested reader are referred to [6, 12, 13, 24, 26, 27].

The rest of this paper is organized as follows. In Section 2, we state some $a$ priori estimates for the bounds on the solution and its derivatives, the use of which will be apparent in the analysis of the numerical methods. We consider the FOFDM of [20] in Section 3 where we present the error estimate before applying Richardson extrapolation on this method. The construction, analysis and Richardson extrapolation of a FMFDM are presented in Section 4. Numerical results to support our theoretical findings are displayed in Section 5. A discussion on the main results of this paper is provided in Section 6 . 


\section{Some a priori estimates for the bounds on the solution and its derivatives}

In this section, we present the bounds on the solution of the problem (1.1)(1.2) and its derivatives.

We shall denote by $\Omega_{l}=[-1,-\delta], \Omega_{c}=[-\delta, \delta], \Omega_{r}=[\delta, 1]$, where $0<$ $\delta \leq \frac{1}{2}$; the left, central and right part of the domain, respectively. Note that $\beta=b(0) / a^{\prime}(0)<0$.

Let $k$ be a positive integer. We define

$$
\begin{aligned}
& S_{1}=\{\|a\|,\|b\|,\|f\|, 1-\delta,|B|, u(\delta), k\}, \\
& S_{2}=\{\|a\|,\|b\|,\|f\|, 1-\delta,|A|, u(-\delta), k\}
\end{aligned}
$$

and

$$
S_{3}=\left\{\|a\|,\|b\|,\|f\|, \beta_{s}, b_{0},|A|,|B|, k\right\} .
$$

Depending on whether $x$ belongs to $\Omega_{l}, \Omega_{c}$ or $\Omega_{r}$, the appropriate bounds are provided in the following lemmas [7].

Lemma 2.1. If $u(x)$ is the solution of the $\operatorname{SPTPP}(1.1)-(1.2)$ and $a, b$ and $f \in C^{k}(\bar{\Omega}), k>0$, then there exist positive constants $\eta$ and $C$ depending only on $S_{1}$ such that

$$
\left|u^{(j)}(x)\right| \leq C\left[1+\varepsilon^{-j} \exp (-2 \eta(1-x) / \varepsilon)\right], j=1,2, \ldots, k+1, x \in \Omega_{r} .
$$

Proof. See [7].

Lemma 2.2. If $u(x)$ is the solution of the $\operatorname{SPTPP}(1.1)-(1.2)$ and $a, b$ and $f \in C^{k}(\bar{\Omega}), k>0$, then there exist positive constants $\eta$ and $C$ depending only on $S_{2}$ such that

$$
\left|u^{(j)}(x)\right| \leq C\left[1+\varepsilon^{-j} \exp (-2 \eta(1+x) / \varepsilon)\right], j=1,2, \ldots, k+1, x \in \Omega_{l} .
$$

Proof. See [7].

Lemma 2.3. If $u(x)$ is the solution of the $\operatorname{SPTPP}(1.1)-(1.2)$ and $a, b$ and $f \in C^{k}(\bar{\Omega}), k>0$, then there exists a positive constant $C$ depending only on $S_{3}$ such that

$$
\left|u^{(j)}\right| \leq C, \quad \forall x \in \Omega_{c}, \quad j=0,1, \ldots, k .
$$

Proof. See [7]

The following lemma gives the bounds on the derivatives of the components of the solution of the SPTPP (1.1)-(1.2).

Lemma 2.4. The solution $u$ of the SPTPP (1.1)-(1.2) can be decomposed as

$$
u=v+w,
$$

where, for all $j, 0 \leq j \leq k$, and all $x \in[-1,1]$, the smooth component $v$ satisfies

$$
\left|v^{(j)}(x)\right| \leq C\left(1+\varepsilon^{-(j-2)} \exp (-2 \eta(1+x) / \varepsilon), \quad x \in[-1,0],\right.
$$




$$
\left|v^{(j)}(x)\right| \leq C\left(1+\varepsilon^{-(j-2)} \exp (-2 \eta(1-x) / \varepsilon), \quad x \in[0,1],\right.
$$

and the singular component $w$ satisfies

$$
\begin{gathered}
\left|w^{(j)}(x)\right| \leq C \varepsilon^{-j} \exp (-2 \eta(1+x) / \varepsilon), \quad x \in[-1,0], \\
\left|w^{(j)}(x)\right| \leq C \varepsilon^{-j} \exp (-2 \eta(1-x) / \varepsilon), \quad x \in[0,1],
\end{gathered}
$$

for some constants $\eta$ and $C$ independent of $\varepsilon$.

Proof. By Lemma 2.3, there exists a constant $D$ such that $u(0)=D$. The SPTPP (1.1)-(1.2) can therefore be regarded as a juxtaposition of two problems, namely:

$$
\varepsilon u^{\prime \prime}+a(x) u^{\prime}-b(x) u=f(x), \quad x \in(0,1), u(0)=D, u(1)=B,
$$

and

$$
\varepsilon u^{\prime \prime}+a(x) u^{\prime}-b(x) u=f(x), \quad x \in(-1,0), \quad u(-1)=A, \quad u(0)=D .
$$

(each of which features like the convection-diffusion problem of [17] at page 53.) We follow [17]. Firstly, let us consider the problem (2.3) which we can write as

$$
-\varepsilon u^{\prime \prime}(x)-a(x) u^{\prime}=g(x), \quad x \in(0,1), u(0)=D, \quad u(1)=B,
$$

where $g(x)=-f(x)-b(x) u$.

The solution $u$ of $(2.5)$ can be written under the form

$$
u=v_{0}+\varepsilon y_{1}+w_{0},
$$

where $v_{0}$ is the solution of the reduced problem

$$
-a(x) v_{0}^{\prime}=g(x), \quad v_{0}(0)=D ;
$$

$y_{1}$ satisfies

$$
L y_{1}=v_{0}^{\prime \prime}, \quad y_{1}(0)=-w_{0}(0) / \varepsilon, \quad y_{1}(1)=0
$$

and $w_{0}$ is the solution of the homogeneous problem

$$
L w_{0}=0 \quad w_{0}=w_{0}(1) \exp (-2 \eta / \varepsilon), \quad w_{0}(1)=B-v_{0}(1) .
$$

It is clear that $\left|w_{0}(0)\right|,\left|w_{0}(1)\right|,\left|y_{1}(0)\right|$ and $\left|v_{0}^{\prime \prime}\right|$ are all bounded by a constant independent of $\varepsilon$. It follows that $y_{1}$ is the solution of a problem similar to (1.1)-(1.2), thus for $j=1,2, \ldots, k+1$,

$$
\left|y_{1}^{(j)}(x)\right| \leq C\left(1+\varepsilon^{-j} \exp (-2 \eta(1-x) / \varepsilon)\right) .
$$

(see bounds in Lemma 2.1.) To obtain bounds on the singular component of the solution $w_{0}$ and its derivatives, we proceed as follows. We define the comparison functions

$$
\Psi^{ \pm}(x)=\left|w_{0}(1)\right| \exp (-2 \eta(1-x) / \varepsilon) \pm w_{0}(x) .
$$


Applying the minimum principle to these functions, we see that $\Psi^{ \pm}(x) \geq 0$ and consequently,

$$
\left|w_{0}(x)\right| \leq C \exp (-2 \eta(1-x) / \varepsilon) \text { for all } x \in[0,1] .
$$

The singular component $w_{0}$ of the solution can be written in the form

$$
w_{0}=w_{0}(0) \varphi+w_{0}(1)(1-\varphi)
$$

where

$$
\varphi(x)=\frac{\int_{x}^{1} \exp (-A(t) / \varepsilon) d t}{\int_{0}^{1} \exp (-A(t) / \varepsilon) d t}
$$

and $A(x)=-\int_{x}^{1} a(s) d s$. And so, $w_{0}^{\prime}=\left(w_{0}(0)-w_{0}(1)\right) \varphi^{\prime}$. Taking into account the bounds on the coefficient $a(x)$ and the fact that

$$
\varphi^{\prime}(x)=\frac{-\exp (-A(x) / \varepsilon)}{\int_{0}^{1} \exp (-A(t) / \varepsilon) d t},
$$

we have

$$
\left|\varphi^{\prime}(x)\right| \leq C \varepsilon^{-1} \exp (-2 \eta(1-x) / \varepsilon)
$$

leading to the bound

$$
\left|w_{0}^{\prime}(x)\right| \leq C \varepsilon^{-1} \exp (-2 \eta(1-x) / \varepsilon)
$$

Since $L w_{0}=0$, the higher order derivatives of $w_{0}$ can be estimated immediately from the estimates of $w_{0}$ and $w_{O}^{\prime}$. Thus, for $0 \leq j \leq k$ :

$$
\left|w_{0}^{(j)}(x)\right| \leq C \varepsilon^{-j} \exp (-2 \eta(1-x) / \varepsilon) .
$$

Since $u^{(j)}=v_{0}^{(j)}+\varepsilon y_{1}^{(j)}+w_{0}^{(j)}$, we have

$$
\left|\left(v_{0}^{(j)}+\varepsilon y_{1}^{(j)}\right)(x)\right| \leq C\left(1+\varepsilon^{-j} \exp (-2 \eta(1-x) / \varepsilon)\right)
$$

and

$$
\left|w_{0}^{(j)}(x)\right| \leq C \varepsilon^{-j} \exp (-2 \eta(1-x) / \varepsilon)
$$

for $0 \leq j \leq k$ and all $x \in[0,1]$.

The component $y_{1}$ can also be decomposed in the same manner as was $u$ : $y_{1}=v_{1}+\varepsilon v_{2}+w_{1}$ where for $0 \leq j \leq k$, and for all $x \in[0,1]$, we have

$$
\begin{gathered}
\left|v_{1}^{(j)}(x)\right| \leq C, \\
\left|v_{2}^{(j)}(x)\right| \leq C\left(1+\varepsilon^{-j} \exp (-2 \eta(1-x) / \varepsilon)\right), \\
\left|w_{1}^{(j)}(x)\right| \leq C \varepsilon^{-j} \exp (-2 \eta(1-x) / \varepsilon) .
\end{gathered}
$$

We combine these two decompositions and obtain:

$$
u=v+w,
$$

where $v=v_{0}+\varepsilon v_{1}+\varepsilon^{2} v_{2}$ and $w=w_{0}+\varepsilon w_{1}$. The components $v$ and $w$ satisfy

$$
\begin{gathered}
L v=f, \quad v(0)=D-w(0), \quad v(1)=B-w(1) ; \\
L w=0, \quad w(0)=w(1) \exp (-2 \eta / \varepsilon),
\end{gathered}
$$


where $w(1)$ is chosen so that the successive derivatives of $v$ (up to order $k$ ) are bounded uniformly in $\varepsilon$.

In virtue of the above, the following estimates hold for the components $v$ and $w$.

$$
\begin{gathered}
\left|v^{(j)}(x)\right| \leq C\left(1+\varepsilon^{-(j-2)} \exp (-2 \eta(1-x) / \varepsilon)\right) \\
\left|w^{(j)}(x)\right| \leq C \varepsilon^{-j} \exp (-2 \eta(1-x) / \varepsilon) .
\end{gathered}
$$

Following the same lines as above, it can be easily shown that the solution $u$ of problem (2.4) can also be written in the form $u=v+w$ where

$$
\begin{gathered}
\left|v^{(j)}(x)\right| \leq C\left(1+\varepsilon^{-(j-2)} \exp (-2 \eta(1+x) / \varepsilon)\right), \\
\left|w^{(j)}(x)\right| \leq C \varepsilon^{-j} \exp (-2 \eta(1+x) / \varepsilon)
\end{gathered}
$$

for all $0 \leq j \leq k$.

\section{Richardson extrapolation on fitted operator finite difference method}

Here we first present the FOFDM which is developed in [20] and the associated error estimates. Then we analyze the effect of Richardson extrapolation on this scheme.

\subsection{The fitted operator finite difference method (FOFDM)}

Let $n$ be a positive and even integer. Consider the following partition of the interval $[-1,1]$ :

$$
x_{0}=-1, x_{j}=x_{0}+j h, j=0,1, \ldots, n-1, h=x_{j}-x_{j-1}, x_{n}=1
$$

and denote the numerical solution on this partition by $U_{j}, j=0,1, \ldots, n$. Also, we denote the above uniform mesh by $\mu_{n}$.

On $\mu_{n}$, the following FOFDM has been designed and analyzed for (1.1)-(1.2) in [20]:

$$
\begin{gathered}
r_{j}^{-} U_{j-1}+r_{j}^{c} U_{j}+r_{j}^{+} U_{j+1}=\tilde{f}_{j}, \quad j=1,2, \ldots, n-1, \\
U_{0}=A, \quad U_{n}=B,
\end{gathered}
$$

where

$$
r_{j}^{+}=\frac{\varepsilon}{\widetilde{\phi}_{j}^{2}}+\frac{\widetilde{a}_{j}}{h}, \quad r_{j}^{c}=-\frac{2 \varepsilon}{\widetilde{\phi}_{j}^{2}}-\frac{\widetilde{a}_{j}}{h}-\widetilde{b}_{j}, \quad r_{j}^{-}=\frac{\varepsilon}{\widetilde{\phi}_{j}^{2}} \text { for } j=1,2, \ldots, \frac{n}{2}-1,
$$

and

$$
r_{j}^{+}=\frac{\varepsilon}{\widetilde{\phi}_{j}^{2}}, \quad r_{j}^{c}=-\frac{2 \varepsilon}{\widetilde{\phi}_{j}^{2}}+\frac{\widetilde{a}_{j}}{h}-\widetilde{b}_{j}, \quad r_{j}^{-}=\frac{\varepsilon}{\widetilde{\phi}_{j}^{2}}-\frac{\widetilde{a}_{j}}{h} \text { for } j=\frac{n}{2}, \frac{n}{2}+1, \ldots, n-1 .
$$


The mesh function $\widetilde{\phi}_{j}$ is given by

$$
\widetilde{\phi}_{j}^{2}= \begin{cases}\frac{h \varepsilon}{\widetilde{a}_{j}}\left(\exp \left(\frac{\widetilde{a}_{j} h}{\varepsilon}\right)-1\right), & j=0,1, \ldots, \frac{n}{2}-1, \\ \frac{h \varepsilon}{\widetilde{a}_{j}}\left(1-\exp \left(-\frac{\widetilde{a}_{j} h}{\varepsilon}\right)\right), & j=\frac{n}{2}+1, \frac{n}{2}+2, \ldots, n, \\ h^{2}, & j=\frac{n}{2},\end{cases}
$$

where

$$
\widetilde{a}_{j}=\frac{a_{j}+a_{j+1}}{2}, \widetilde{b}_{j}=\frac{b_{j-1}+b_{j}+b_{j+1}}{3} \text { and } \tilde{f}_{j}=\frac{f_{j-1}+f_{j}+f_{j+1}}{3} .
$$

The following theorem regarding FOFDM (3.7)-(3.8) was proved in [20]:

Theorem 3.1. Let $a(x), b(x)$ and $f(x)$ be sufficiently smooth functions in the problem (1.1)-(1.2) and so that $u(x) \in C^{4}([-1,1])$. The numerical solution $U$ obtained via the FOFDM (3.7)-(3.8) satisfies the following estimate:

$$
\sup _{0<\varepsilon \leq 1} \max _{0 \leq j \leq n}\left|u_{j}-U_{j}\right| \leq M h .
$$

Hereafter, $M$ denotes a positive constant which may take different values in different equations/inequalities but is always independent of $h$ and $\varepsilon$.

In this paper we would like to see whether Richardson extrapolation can improve the estimate provided in (3.10) above.

\subsection{Richardson extrapolation on FOFDM}

Richardson extrapolation consists of taking a linear combination of $k$ solutions $(k \geq 2)$ corresponding to different but nested meshes on the intersection of these meshes which is in fact the coarsest mesh [9]. In order to achieve better accuracy, we compute two numerical solutions via our FOFDM on two but nested meshes. We show that with a suitable choice of a linear combination of these two solutions, one can improve the accuracy and the order of convergence $[6,27]$.

To begin with, let us denote by $\mu_{2 n}$ the mesh obtained by bisecting each mesh interval in $\mu_{n}$, i.e.,

$\mu_{2 n}=\left\{\bar{x}_{j}\right\}$ with $\bar{x}_{0}=-1, \bar{x}_{n}=1$ and $\bar{x}_{j}-\bar{x}_{j-1}=\bar{h}=h / 2, \quad j=1,2, \ldots, 2 n$.

We denote the analytical and numerical solutions on the mesh $\mu_{2 n}$ by $\bar{u}_{j}$ and $\bar{U}_{j}$, respectively.

From estimate (3.10), we have on one hand

$$
u_{j}-U_{j}=M h+R_{n}\left(x_{j}\right), \quad 1 \leq j \leq n-1 .
$$

On the other hand, we have

$$
\bar{u}_{j}-\bar{U}_{j}=M \bar{h}+R_{2 n}\left(\bar{x}_{j}\right), \quad 1 \leq j \leq 2 n-1 .
$$

Therefore,

Let

$$
u_{j}-\left(2 \bar{U}_{j}-U_{j}\right)=o(h), \quad \forall 1 \leq j \leq n-1
$$

$$
U_{j}^{\text {ext }}:=2 \bar{U}_{j}-U_{j} \text {. }
$$


Thus $U_{j}^{\text {ext }}$ is another numerical approximation of $u_{j}$.

Now, for $j=1,2, \ldots, n / 2-1$ we have

$$
\begin{aligned}
L^{h}\left(u_{j}-U_{j}\right) & =\left(r_{j}^{-} u_{j-1}+r_{j}^{c} u_{j}+r_{j}^{+} u_{j+1}\right)-\widetilde{f}_{j} \\
& =T_{0} u_{j}+T_{1} u_{j}^{\prime}+T_{2} u_{j}^{\prime \prime}+T_{3} u_{j}^{\prime \prime \prime}+T_{4} u^{(i v)}\left(\xi_{j}\right),
\end{aligned}
$$

where $\xi_{j} \in\left(x_{j-1}, x_{j+1}\right)$ and

$$
\begin{aligned}
& T_{0}=r_{j}^{-}+r_{j}^{c}+r_{j}^{+}+b_{j}+\frac{h^{2}}{3} b_{j}^{\prime \prime}, \\
& T_{1}=h\left(r_{j}^{+}-r_{j}^{-}\right)-a_{j}-\frac{h^{2}}{3}\left(a_{j}^{\prime \prime}-2 b_{j}^{\prime}\right), \\
& T_{2}=\frac{h^{2}}{2}\left(r_{j}^{+}+r_{j}^{-}\right)-\varepsilon-\frac{h^{2}}{3}\left(2 a_{j}^{\prime}-b_{j}-\frac{h^{2}}{2} b_{j}^{\prime \prime}\right), \\
& T_{3}=\frac{h^{3}}{6}\left(r_{j}^{+}-r_{j}^{-}\right)-\frac{h^{2}}{3}\left(a_{j}+\frac{h^{2}}{2} a_{j}^{\prime \prime}\right), \\
& T_{4}=\frac{h^{4}}{24}\left(r_{j}^{+}+r_{j}^{-}\right)-\frac{h^{2}}{3} \varepsilon .
\end{aligned}
$$

Similarly, for $j=1(1) n / 2-1$, we have

$$
L^{\bar{h}}\left(u_{j}-\bar{U}_{j}\right)=\bar{T}_{0} u_{j}+\bar{T}_{1} u_{j}^{\prime}+\bar{T}_{2} u_{j}^{\prime \prime}+\bar{T}_{3} u_{j}^{\prime \prime \prime}+\bar{T}_{4} u^{(i v)}\left(\bar{\xi}_{j}\right),
$$

where $\bar{\xi}_{j} \in\left(\left(x_{j-1}+x_{j}\right) / 2,\left(x_{j}+x_{j+1}\right) / 2\right)$ and the $\bar{T}$ s are obtained from the $T \mathrm{~s}$ by substituting $h$ by $\bar{h}$.

Some algebraic manipulations yield

$$
\left|L^{h}\left(u_{j}-U_{j}\right)\right| \leq M h,
$$

and

The inequality

$$
\left|L^{\bar{h}}\left(u_{j}-\bar{U}_{j}\right)\right| \leq M h .
$$

$$
\left|L^{h}\left(u_{j}-U_{j}^{e x t}\right)\right| \leq\left|L^{h}\left(u_{j}-U_{j}\right)\right|+2\left|L^{\bar{h}}\left(u_{j}-\bar{U}_{j}\right)\right|
$$

leads to

$$
\left|L^{h}\left(u_{j}-U_{j}^{e x t}\right)\right| \leq M h .
$$

The following lemmas (see [20] for the proof) are required in this analysis.

Lemma 3.2 (Discrete minimum principle). For any mesh function $\xi_{j}$ such that $L^{h} \xi_{j} \leq 0, \forall j=1,2, \ldots, n-1, \xi_{0} \geq 0$ and $\xi_{n} \geq 0$, we have $\xi_{j} \geq 0, \forall j=$ $0,1, \ldots, n$.

Lemma 3.3. If $Z_{i}$ is any mesh function such that $Z_{0}=Z_{n}=0$, then there exists a positive constant $\eta$ such that

$$
\left|Z_{i}\right| \leq \frac{1}{\eta} \max _{1 \leq j \leq n-1}\left|L^{h} Z_{j}\right| \text { for } 0 \leq i \leq n
$$


Using the lemma above, equation (3.13) leads to

$$
\left|u_{j}-U_{j}^{\text {ext }}\right| \leq M h \text { for all } j=1,2, \ldots, \frac{n}{2}-1 .
$$

Following the same procedure, it is easy to see that

$$
\left|u_{j}-U_{j}^{\text {ext }}\right| \leq M h \text { for all } j=\frac{n}{2}, \frac{n}{2}+1, \ldots, n-1 .
$$

Consequently, combining equations (3.14) and (3.15), we obtain the following result.

Theorem 3.4. Let $a(x), b(x)$ and $f(x)$ be sufficiently smooth functions in the problem (1.1)-(1.2) and so that $u(x) \in C^{4}([-1,1])$. Then the numerical solution $U^{\text {ext }}$ obtained via Richardson extrapolation based on FOFDM (3.7) along with (3.8) satisfies the following estimate:

$$
\sup _{0<\varepsilon \leq 1} \max _{1 \leq j \leq n-1}\left|u_{j}-U_{j}^{\text {ext }}\right| \leq M h .
$$

\section{Richardson extrapolation on fitted mesh finite difference method}

The idea from Chapter 8 of Miller et al. [17] is used in this section to develop a fitted mesh finite difference scheme. The convergence of the scheme is analyzed before embarking on the study of the effect of Richardson extrapolation on its accuracy and rate of convergence.

\subsection{The fitted mesh finite difference method (FMFDM)}

It is assumed that there are two boundary layers, one at each end, and let the interval $[-1,1]$ be partitioned as

$$
[-1,1]:=[-1,-1+\tau] \cup[-1+\tau, 1-\tau] \cup[1-\tau, 1],
$$

where $\tau$ denotes the mesh transition parameter.

Let $n$ be a positive integer such that $n=2^{m}$ with $m \geq 3$.

To construct the piece-wise uniform mesh (of Shishkin type), we subdivide both intervals $[-1,-1+\tau]$ and $[1-\tau, 1]$ into $n / 4$ equal mesh elements while we subdivide the interval $[-1+\tau, 1-\tau]$ into $n / 2$ equal mesh elements. This gives

$$
\begin{aligned}
{[-1,1]:=-1 } & =x_{0}<x_{1}<\cdots<x_{n / 4}<\cdots<x_{n / 2} \\
& =0<\cdots<x_{3 n / 4}<\cdots<x_{n}=1 .
\end{aligned}
$$

The parameter $\tau$ is defined by

$$
\tau=\min \left\{\frac{1}{4}, \frac{\varepsilon}{\eta} \ln \left(\frac{n}{4}\right)\right\},
$$

where $\eta$ is a positive constant. The mesh spacing $h_{j}=x_{j}-x_{j-1}$ is given by

$$
h_{j}= \begin{cases}4 \tau n^{-1}, & j=1,2, \ldots, n / 4,3 n / 4+1,3 n / 4+2, \ldots, n-1, n \\ 4(1-\tau) n^{-1}, & j=n / 4+1, n / 4+2, \ldots, 3 n / 4 .\end{cases}
$$


We denote this mesh by $\mu_{n, \tau}$.

Using the above conventions, we discretize the problem (1.1)-(1.2) on $\mu_{n, \tau}$ as

$$
\begin{cases}\varepsilon \widetilde{D} U_{j}+a_{j} D^{+} U_{j}-b_{j} U_{j}=f_{j}, & a_{j}>0 ; \\ \varepsilon \widetilde{D} U_{j}+a_{j} D^{-} U_{j}-b_{j} U_{j}=f_{j}, & a_{j} \leq 0\end{cases}
$$

$$
U_{0}=A, \quad U_{n}=B,
$$

where

$$
\begin{aligned}
& D^{+} U_{j}=\frac{U_{j+1}-U_{j}}{h_{j+1}}, \\
& D^{-} U_{j}=\frac{U_{j}-U_{j-1}}{h_{j}},
\end{aligned}
$$

and

$$
\widetilde{D} U_{j}=\frac{2}{h_{j}+h_{j+1}}\left(D^{+} U_{j}-D^{-} U_{j}\right) .
$$

Equations (4.19) can be written in the form

$$
L^{n}\left(U_{j}\right):=r_{j}^{-} U_{j-1}+r_{j}^{c} U_{j}+r_{j}^{+} U_{j+1}=f_{j}, \quad j=1,2, \ldots, n-1,
$$

where, for $j=1,2, \ldots, \frac{n}{2}-1$, we have

$$
\begin{gathered}
r_{j}^{+}=\frac{2 \varepsilon}{h_{j+1}\left(h_{j+1}+h_{j}\right)}+\frac{a_{j}}{h_{j+1}}, \\
r_{j}^{c}=-\frac{2 \varepsilon}{h_{j} h_{j+1}}-\frac{a_{j}}{h_{j+1}}-b_{j}, \\
r_{j}^{-}=\frac{2 \varepsilon}{h_{j}\left(h_{j}+h_{j+1}\right)},
\end{gathered}
$$

and for $j=\frac{n}{2}, \frac{n}{2}+1, \ldots, n-1$, we have

$$
\begin{gathered}
r_{j}^{+}=\frac{2 \varepsilon}{h_{j+1}\left(h_{j+1}+h_{j}\right)}, \\
r_{j}^{c}=-\frac{2 \varepsilon}{h_{j} h_{j+1}}+\frac{a_{j}}{h_{j}}-b_{j}, \\
r_{j}^{-}=\frac{2 \varepsilon}{h_{j}\left(h_{j}+h_{j+1}\right)}-\frac{a_{j}}{h_{j}} .
\end{gathered}
$$

The discrete operator $L^{n}$ satisfies the following minimum principle:

Lemma 4.1. For any mesh function $\xi_{j}$ such that $L^{n} \xi_{j} \leq 0, \forall j=1,2, \ldots, n-1$, $\xi_{0} \geq 0$ and $\xi_{n} \geq 0$, we have $\xi_{j} \geq 0, \forall j=0,1, \ldots, n$. 
Proof. Let $k$ be such that $\xi_{k}=\min _{0 \leq j \leq n} \xi_{j}$ and suppose that $\xi_{k}<0$. It's clear that $k \notin\{0, n\}$. Also $\xi_{k+1}-\xi_{k} \geq 0$, and $\xi_{k}-\xi_{k-1} \leq 0$.

On one hand we have

$$
L^{n} \xi_{k}=\varepsilon \widetilde{D} \xi_{k}+a_{k} D^{+} \xi_{k}-b_{k} \xi_{k}>0, \quad 1 \leq k \leq n / 2-1 .
$$

On the other hand

$$
L^{n} \xi_{k}=\varepsilon \widetilde{D} \xi_{k}+a_{k} D^{-} \xi_{k}-b_{k} \xi_{k}>0, \quad n / 2 \leq k \leq n-1 .
$$

Thus $L^{n} \xi_{k}>0,1 \leq k \leq n-1$, which is a contradiction. It follows that $\xi_{k} \geq 0$ and thus $\xi_{j} \geq 0,0 \leq j \leq n$.

This minimum principle is used to prove the following lemma.

Lemma 4.2. If $Z_{i}$ is any mesh function such that $Z_{0}=Z_{n}=0$, then

$$
\left|Z_{i}\right| \leq \frac{1}{\eta} \max _{1 \leq j \leq n-1}\left|L^{n} Z_{j}\right| \text { for } 0 \leq i \leq n .
$$

Proof. Let us define two comparison functions $Y_{i}^{ \pm}$by

$$
Y_{i}^{ \pm}=\frac{x_{i}}{a^{*}} \max _{1 \leq j \leq n-1}\left|L^{n} Z_{j}\right| \pm Z_{i}, \quad 0 \leq i \leq n,
$$

where

$$
a^{*}= \begin{cases}-\eta & \text { if } 0 \leq i \leq n / 2-1 \\ \eta & \text { if } n / 2 \leq i \leq n\end{cases}
$$

It is clear that $Y_{0}^{ \pm} \geq 0$ and $Y_{n}^{ \pm} \geq 0$. Also, observe that

$$
L^{n} Y_{i}^{ \pm}=\frac{a_{i}-b_{i} x_{i}}{a^{*}} \max _{1 \leq j \leq n-1}\left|L^{n} Z_{j}\right| \pm L^{n} Z_{i}, \quad 0 \leq i \leq n .
$$

If $0 \leq i \leq n / 2-1$, then $\left(a_{i}-b_{i} x_{i}\right) /(-\eta) \leq-1$. Likewise, if $n / 2 \leq i \leq n$, then $a_{i}-b_{i} x_{i} / \eta \leq-1$. In either case, $L^{n} Y_{i}^{ \pm} \leq 0$. By the discrete minimum principle (Lemma 4.1), we conclude that $Y_{i} \geq 0, \forall 0 \leq i \leq n$ and this completes the proof.

\section{Convergence analysis of FMFDM}

The restrictions of problem (1.1)-(1.2) to the intervals $[0,1]$ and $[-1,0]$ feature like the convection-diffusion problem considered in Chapter 8 in [17]. In our analysis, we will implement the ideas provided in this work, for the interval $[0,1]$. The analysis on $[-1,0]$ follows similar steps.

We decompose the solution $U$ of the discrete problem (4.19)-(4.20) in its regular part $V$ and singular part $W$. The components $V$ and $W$ of $U$ are solutions of the problems

$$
L^{n} V=f, \quad V(-1)=v(-1), V(1)=v(1)
$$

and

respectively.

$$
L^{n} W=0, W(-1)=w(-1), W(1)=w(1),
$$


We can write the error in the form

$$
U-u=(V-v)+(W-w)
$$

and estimate the components of the error separately.

We start with the regular component.

The local truncation error is given by

$$
L^{n}(V-v)=\varepsilon\left(\frac{d^{2}}{d x^{2}}-\widetilde{D}\right) v+a\left(\frac{d}{d x}-D^{-}\right) v .
$$

Using Lemma 1 (p. 21 of [17]), we obtain

$$
\begin{aligned}
\left|L^{n}\left(V_{j}-v_{j}\right)\right| \leq & \frac{\varepsilon}{3}\left(x_{j+1}-x_{j-1}\right)\left|v_{j}^{\prime \prime \prime}\right| \\
& +\frac{a_{j}}{2}\left(x_{j}-x_{j-1}\right)\left|v_{j}^{\prime \prime}\right| \text { for } \frac{n}{2} \leq j \leq n-1 .
\end{aligned}
$$

Since $h_{j}=x_{j}-x_{j-1} \leq 4 n^{-1}$ for any $j$, therefore using Lemma 2.4, we obtain

$$
\left|L^{n}\left(V_{j}-v_{j}\right)\right| \leq M n^{-1} \text {. }
$$

Hence, by Lemma 4.2

$$
\left|V_{j}-v_{j}\right| \leq M n^{-1}
$$

The estimate on $L^{n}(W-w)$ depends on whether $\tau=1 / 4$ or $\tau=(\varepsilon / \eta) \ln (n / 4)$.

If $\tau=1 / 4$, the mesh is uniform and $1 / 4 \leq(\varepsilon / \eta) \ln (n / 4)$. The local truncation error $L^{n}(W-w)$ is given by

$$
\begin{aligned}
\left|L^{n}\left(W_{j}-w_{j}\right)\right| \leq & \frac{\varepsilon}{3}\left(x_{j+1}-x_{j-1}\right)\left|w_{j}^{\prime \prime \prime}\right| \\
& +\frac{a_{j}}{2}\left(x_{j}-x_{j-1}\right)\left|w_{j}^{\prime \prime}\right| \text { for } \frac{n}{2} \leq j \leq n-1 .
\end{aligned}
$$

By Lemma 2.4 and the fact that $h_{j}=x_{j}-x_{j-1}=4 n^{-1}$, the above inequality gives

$$
\left|L^{n}\left(W_{j}-w_{j}\right)\right| \leq M \varepsilon^{-2} n^{-1} .
$$

Now since $\varepsilon^{-1}$ is less than $(4 / \eta) \ln (n / 4)$, we have

$$
\left|L^{n}\left(W_{j}-w_{j}\right)\right| \leq M n^{-1}(\ln (n / 4))^{2} .
$$

Using Lemma 4.2 then we obtain

$$
\left|W_{j}-w_{j}\right| \leq M n^{-1}(\ln (n / 4))^{2} .
$$

If $\tau=(\varepsilon / \eta) \ln (n / 4)$, then the mesh is piecewise uniform. In each of the subintervals $[0,1-\tau]$ and $[1-\tau, 1]$, a different argument is used to bound $W-w$.

Both $W$ and $w$ are small on the subinterval with no boundary layer, namely $[0,1-\tau]$. Therefore, since $|W-w| \leq|W|+|w|$, we will bound $W$ and $w$ separately. Before we move any further, let us note that $w$ can also be decomposed 
as $w=w_{0}+\varepsilon w_{1}$ (see [17], p. 59). Introducing the function $\varphi$ by

$$
\varphi(x)=\frac{\int_{x}^{1} \exp (-A(t) / \varepsilon) d t}{\int_{0}^{1} \exp (-A(t) / \varepsilon) d t}, \quad A(t)=\int_{x}^{1} a(s) d s .
$$

It can be shown that $w_{0}$ can be written in the form

$$
w_{0}(x)=w_{0}(0) \varphi(x)+w_{0}(1)(1-\varphi(x))
$$

and therefore

$$
w_{0}^{\prime}(x)=\left(w_{0}(0)-w_{0}(1)\right) \varphi^{\prime}(x) .
$$

But $w_{0}(0)=w_{0}(1) \exp (-\eta / \varepsilon)$ and hence

$$
\frac{w_{0}^{\prime}(x)}{w_{0}(1)}=-\left(1-\exp (-\eta / \varepsilon) \varphi^{\prime}(x)>0 .\right.
$$

It follows that $w_{0}(x) / w_{0}(1)$ is positive and increasing in the interval $[0,1]$.

Thus

$$
0 \leq \frac{w_{0}(x)}{w_{0}(1)} \leq \frac{w_{0}(1-\tau)}{w_{0}(1)}
$$

and hence

$$
\left|w_{0}(x)\right| \leq\left|w_{0}(1-\tau)\right|, \quad \forall x \in[0,1-\tau] .
$$

The same is true for $w_{1}(x)$ and since $w=w_{0}+\varepsilon w_{1}$, it follows that

$$
|w(x)| \leq|w(1-\tau)|, \quad \forall x \in[0,1-\tau] .
$$

Using the estimates for $w_{0}, w_{1}$, given that $w_{0}(1-\tau)=w_{0} \exp (\eta(1-\tau) / \varepsilon)$, and the fact that $\tau=(\varepsilon / \eta) \ln (n / 4)$, we obtain

$$
|w(x)| \leq M \exp (-\eta \tau / \varepsilon)=M n^{-1} .
$$

Now we define an auxiliary mesh function $\widetilde{W}$ analogous to $W$ except that the coefficient $a$ in the difference operator $L^{n}$ is replaced by $\eta$. Then Lemma 5 on p. 53 of [17] suggests that

$$
\left|W_{j}\right| \leq\left|\widetilde{W}_{j}\right|, \quad \forall 0 \leq j \leq n .
$$

Thus by Lemma 3 (p. 51 of [17]), we conclude that

$$
\left|W_{j}\right| \leq M n^{-1} \text { for } n / 2 \leq j \leq 3 n / 4 \text {. }
$$

Hence, from inequalities (4.28) and (4.29), we have

$$
\left|W_{j}-w_{j}\right| \leq M n^{-1} \text { for } n / 2 \leq j \leq 3 n / 4 .
$$

In the subinterval $[1-\tau, 1]$, an argument analogue to (4.25) leads to

$$
\left|L^{n}\left(W_{j}-w_{j}\right)\right| \leq M \varepsilon^{-2}\left|x_{j+1}-x_{j-1}\right|=8 M \varepsilon^{-2} \tau n^{-1} .
$$

But also, we have

$$
\left|W_{n}-w_{n}\right|=0
$$

and from the inequality (4.30)

$$
\left|W_{3 n / 4}-w_{3 n / 4}\right| \leq\left|W_{3 n / 4}\right|+\left|w_{3 n / 4}\right| \leq M n^{-1} .
$$


By introducing the barrier function

$$
\Phi_{j}=\left(x_{j}-(1-\tau)\right) M_{1} \varepsilon^{-2} \tau n^{-1}+M_{2} n^{-1},
$$

we see that the mesh functions

$$
\Psi_{j}^{ \pm}=\Phi_{j} \pm\left(W_{j}-w_{j}\right)
$$

satisfy

$$
\Psi_{3 n / 4}^{ \pm} \geq 0, \quad \Psi_{n}^{ \pm}=0
$$

provided that the constants $M_{1}$ and $M_{2}$ are chosen suitably.

Note that

$$
L^{n} \Psi_{j}^{ \pm} \leq 0,3 n / 4+1 \leq j \leq n-1 .
$$

By the discrete minimum principle (Lemma 3.2 ) on $[1-\tau, 1]$ we get

$$
\Psi_{j}^{ \pm} \geq 0,3 n / 4 \leq j \leq n .
$$

Consequently,

$$
\left|W_{j}-w_{j}\right| \leq \Phi_{j} \leq M_{1} \varepsilon^{-2} \tau^{2} n^{-1}+M_{2} n^{-1},
$$

and making use of the inequality $\tau \leq(\varepsilon / \eta) \ln (n / 4)$, we obtain

$$
\left|W_{j}-w_{j}\right| \leq M n^{-1}(\ln (n / 4))^{2} .
$$

Combining (4.30) and (4.31), we obtain the following estimate on the singular component of the error over the interval $[0,1]$ :

$$
\left|W_{j}-w_{j}\right| \leq M n^{-1}(\ln (n / 4))^{2}, \quad n / 2 \leq j \leq n .
$$

Estimates (4.24) and (4.32) along with the inequality (4.22) immediately gives

$$
\left|U_{j}-u_{j}\right| \leq M n^{-1}(\ln (n / 4))^{2}, \quad n / 2 \leq j \leq n .
$$

Similarly,

$$
\left|U_{j}-u_{j}\right| \leq M n^{-1}(\ln (n / 4))^{2}, \quad 0 \leq j \leq n / 2-1 .
$$

We therefore have the following result.

Theorem 4.3. Let $a(x), b(x)$ and $f(x)$ be sufficiently smooth functions in the problem (1.1)-(1.2) and so that $u(x) \in C^{4}([-1,1])$. The numerical solution $U$ obtained via FMFDM (4.21) along with (4.20) satisfies

$$
\sup _{0<\varepsilon \leq 1} \max _{0 \leq j \leq n}\left|u_{j}-U_{j}\right| \leq M n^{-1}(\ln (n / 4))^{2} .
$$




\subsection{Richardson extrapolation for FMFDM}

We bisect each mesh sub-interval of $\mu_{n, \tau}$ and obtain a new mesh which we denote by $\mu_{2 n, \tau}$.

$$
\mu_{2 n, \tau}=\left\{\bar{x}_{j}, 0 \leq j \leq 2 n+1\right\} \supset \mu_{n, \tau} \text { and } \bar{x}_{j}-\bar{x}_{j-1}=\bar{h}_{j}=h_{j} / 2 .
$$

We denote the numerical solution computed on the mesh $\mu_{2 n, \tau}$ by $\widetilde{U}$.

From the estimate (4.35), we have

$$
u_{j}-U_{j}=M n^{-1}(\ln (n / 4))^{2}+R_{n}\left(x_{j}\right), \forall x_{j} \in \mu_{n, \tau}
$$

and

$$
u_{j}-\widetilde{U}_{j}=M(2 n)^{-1}(\ln (n / 4))^{2}+R_{2 n}\left(\bar{x}_{j}\right), \forall \bar{x}_{j} \in \mu_{2 n, \tau} .
$$

The remainders $R_{n}\left(x_{j}\right)$ and $R_{2 n}\left(\bar{x}_{j}\right)$ are of $O\left(n^{-1}(\ln (n / 4))^{2}\right)$. It is to be noted that in practice, we assume

$$
\tau=\frac{\varepsilon}{\eta} \ln \left(\frac{n}{4}\right),
$$

because the possibility $\tau=1 / 4$ suggested in equation (4.17) means that $1 / 4<$ $(\varepsilon / \eta) \ln (n / 4)$, and so $n^{-1}$ is very small relative to $\varepsilon$. This unlikely situation can be dealt with using the standard techniques.

The presence of the factor $\ln (n / 4)$ in both (4.36) and (4.37) explains the fact that the two meshes $\mu_{n, \tau}$ and $\mu_{2 n, \tau}$ use the same parameter $\tau$ given by (4.38).

A combination of equations (4.36) and (4.37) suggests that

$$
u_{j}-\left(2 \widetilde{U}_{j}-U_{j}\right)=O\left(n^{-1}(\ln (n / 4))^{2}\right), \quad \forall j=1, \ldots, n-1
$$

and therefore we set

$$
U_{j}^{\text {ext }}:=2 \widetilde{U}_{j}-U_{j}, \forall j=1, \ldots, n-1,
$$

as the numerical approximation of $u$ at the grid point $x_{j} \in \mu_{n, \tau}$ resulting from the extrapolation process.

The decomposition of the error after extrapolation in a similar manner as in (4.22) gives

$$
U^{\text {ext }}-u=\left(V^{\text {ext }}-v\right)+\left(W^{\text {ext }}-w\right),
$$

where $V^{\text {ext }}$ and $W^{\text {ext }}$ are the regular and singular components of $U^{\text {ext }}$, respectively. We will estimate the components of the error separately.

For the reasons mentioned in the previous subsection, we will provide the analysis only on the interval $[0,1]$.

The local truncation error of the scheme (4.21) along with (4.20) at the grid point $x_{j}$ after extrapolation is given by

$$
\begin{aligned}
L^{n}\left(u-U^{\mathrm{ext}}\right)= & {\left[2 L_{*}^{n}\left(v_{j}-\widetilde{V}_{j}\right)-L^{n}\left(v_{j}-V_{j}\right)\right] } \\
& +\left[2 L_{*}^{n}\left(w_{j}-\widetilde{W}_{j}\right)-L^{n}\left(w_{j}-W_{j}\right)\right],
\end{aligned}
$$


where, like $L^{n}, L_{*}^{n}$ is a discrete operator associated with (4.21) along with (4.20) but on the mesh $\mu_{2 n, \tau}$.

For the regular part of the local truncation after extrapolation, we use Lemma 1 (p. 21 of [17]). An analogous result as in (4.23) is

$$
\begin{aligned}
\left|2 L_{*}^{n}\left(v_{j}-\widetilde{V}_{j}\right)-L^{n}\left(v_{j}-V_{j}\right)\right| \leq & \frac{2 \varepsilon}{3}\left(x_{j+1 / 2}-x_{j-1 / 2}\right)\left|v_{j}^{\prime \prime}\right|+a_{j}\left(x_{j}-x_{j-1 / 2}\right)\left|v_{j}^{\prime}\right| \\
& +\frac{\varepsilon}{3}\left(x_{j+1}-x_{j-1}\right)\left|v_{j}^{\prime \prime \prime}\right|+\frac{a_{j}}{2}\left(x_{j}-x_{j-1}\right)\left|v_{j}^{\prime \prime}\right| \\
& \text { for } \frac{n}{2} \leq j \leq n-1 .
\end{aligned}
$$

Using Lemma 3.3, we therefore have

$$
\left|v_{j}-V_{j}^{\mathrm{ext}}\right| \leq M n^{-1} \text { for } \frac{n}{2} \leq j \leq n-1 .
$$

For the estimates on $w_{j}-W_{j}^{\text {ext }}$, we discuss two different cases.

If $\tau=1 / 4$, the mesh is uniform and we have $\varepsilon^{-1} \leq(4 / \eta) \ln (n / 4)$. Therefore, by Lemma 4.21 , we have

$$
\left|2 L_{*}^{n}\left(w_{j}-\widetilde{W}_{j}\right)-L^{n}\left(w_{j}-W_{j}\right)\right| \leq M n^{-1} \varepsilon^{-2} \leq M n^{-1}(\ln (n / 4))^{2} .
$$

An application of Lemma 3.3 then gives

$$
\left|w_{j}-W_{j}^{\text {ext }}\right| \leq M n^{-1}(\ln (n / 4))^{2} \text { for } \frac{n}{2} \leq j \leq n-1 .
$$

If $\tau=(\varepsilon / \eta) \ln (n / 4)$, the mesh is piecewise uniform with mesh spacing of $4(1-$ $\tau) / n$ in the interval $[0,1-\tau]$ and $4 \tau / n$ in the interval $[1-\tau, 1]$.

In the subinterval $[0,1-\tau]$, the functions $w, W$ and $\widetilde{W}$ are small and therefore we have

$$
\left|w_{j}-W_{j}^{\text {ext }}\right| \leq|w|+2|\widetilde{W}|+|W|
$$

The bounds on $|w|$ and $|W|$ are obtained in the previous subsection. Also, bounds of $|W|$ are those of $|\widetilde{W}|$. Hence,

$$
\left|w_{j}-W_{j}^{\mathrm{ext}}\right| \leq M n^{-1} \text { for } \frac{n}{2} \leq j \leq \frac{3 n}{4} .
$$

In the subinterval $[1-\tau, 1]$, we use the discrete minimum principle (Lemma $3.2)$ to bound $\left|w_{j}-W_{j}^{\text {ext }}\right|$. For $3 n / 4+1 \leq j \leq n-1$, we have

$$
L^{n}\left(w_{j}-W_{j}^{\text {ext }}\right) \leq M \varepsilon^{-2}\left|x_{j+1}-x_{j-1}\right|=M \varepsilon^{-2} \tau n^{-1} .
$$

Furthermore, $\left|w_{3 n / 4}-W_{3 n / 4}^{\text {ext }}\right| \leq M n^{-1}$ and $\left|w_{n}-W_{n}^{\text {ext }}\right|=0$.

Defining the barrier function

$$
\bar{\Phi}_{j}=\left(x_{j}-(1-\tau)\right) M_{1} \varepsilon^{-2} \tau n^{-1}+M_{2} n^{-1},
$$

we notice that, for a suitable choice of $M_{1}$ and $M_{2}$, the mesh function

$$
\bar{\Psi}_{j}^{ \pm}=\bar{\Phi}_{j} \pm\left(w_{j}-W_{j}^{\mathrm{ext}}\right)
$$


satisfies

$$
\bar{\Psi}_{3 n / 4}^{ \pm} \geq 0, \quad \bar{\Psi}_{n}^{ \pm}=0
$$

and

$$
L^{n} \bar{\Psi}_{j}^{ \pm} \leq 0 \text { for } \frac{3 n}{4}+1 \leq j \leq n-1 .
$$

It follows, by the discrete minimum principle (Lemma 3.2) that on the interval $[1-\tau, 1]$

$$
\bar{\Psi}_{j}^{ \pm} \geq 0 \text { for } \frac{3 n}{4}+1 \leq j \leq n-1
$$

Therefore

$$
\left|w_{j}-W_{j}^{\text {ext }}\right| \leq \bar{\Phi}_{j} \leq M_{1} \varepsilon^{-2} \tau^{2} n^{-1}+M_{2} n^{-1} .
$$

Hence

$$
\left|w_{j}-W_{j}^{\text {ext }}\right| \leq M n^{-1}(\ln (n / 4))^{2} \text { for } \frac{3 n}{4}+1 \leq j \leq n-1 .
$$

Combining estimates (4.44) and (4.45), we obtain

$$
\left|w_{j}-W_{j}^{\text {ext }}\right| \leq M n^{-1}(\ln (n / 4))^{2} \text { for } \frac{n}{2} \leq j \leq n .
$$

By virtue of (4.40), estimates (4.42) and (4.46) lead to

$$
\left|u_{j}-U_{j}^{\text {ext }}\right| \leq M n^{-1}(\ln (n / 4))^{2} \text { for } \frac{n}{2} \leq j \leq n .
$$

Following the similar lines on the interval $[-1,0]$, i.e., when $a>0$, we obtain the same estimates.

Combining the two, we have:

Theorem 4.4. Let $a(x), b(x)$ and $f(x)$ be sufficiently smooth functions in the problem (1.1)-(1.2) and so that $u(x) \in C^{4}([-1,1])$. The numerical solution $U^{\text {ext }}$ obtained via FMFDM (4.21) along with (4.20) after extrapolation satisfies

$$
\sup _{0<\varepsilon \leq 1} \max _{0 \leq j \leq n}\left|u_{j}-U_{j}^{\text {ext }}\right| \leq M n^{-1}(\ln (n / 4))^{2} .
$$

In next section, we provide test examples to support these theoretical estimates.

\section{Numerical results}

For the following two test examples we provide comparative numerical results before and after extrapolation using the two fitted methods.

Example 5.1 ([14]). Consider problem (1.1)-(1.2) with

$$
a(x)=2(1-2 x), \quad b(x)=4, \quad f(x)=0
$$

for $0<x<1$.

The exact solution is

$$
u(x)=\exp \left(-2 x \frac{1-x}{\varepsilon}\right) .
$$

The solution to this problem has a turning point at $x=0.5$. 
TABle 1. Results for Example 5.2: Maximum errors via FOFDM (3.7) along with (3.8) before extrapolation.

\begin{tabular}{|c|ccccccc|}
\hline$\varepsilon$ & $n=16$ & $n=32$ & $n=64$ & $n=128$ & $n=256$ & $n=512$ & $n=1024$ \\
\hline $1.0 \mathrm{E}-04$ & $3.60 \mathrm{E}-02$ & $1.45 \mathrm{E}-02$ & $6.37 \mathrm{E}-03$ & $2.99 \mathrm{E}-03$ & $1.45 \mathrm{E}-03$ & $7.12 \mathrm{E}-04$ & $3.53 \mathrm{E}-04$ \\
$1.0 \mathrm{E}-05$ & $3.60 \mathrm{E}-02$ & $1.45 \mathrm{E}-02$ & $6.37 \mathrm{E}-03$ & $2.99 \mathrm{E}-03$ & $1.45 \mathrm{E}-03$ & $7.12 \mathrm{E}-04$ & $3.53 \mathrm{E}-04$ \\
$1.0 \mathrm{E}-06$ & $3.60 \mathrm{E}-02$ & $1.45 \mathrm{E}-02$ & $6.37 \mathrm{E}-03$ & $2.99 \mathrm{E}-03$ & $1.45 \mathrm{E}-03$ & $7.12 \mathrm{E}-04$ & $3.53 \mathrm{E}-04$ \\
$1.0 \mathrm{E}-08$ & $3.60 \mathrm{E}-02$ & $1.45 \mathrm{E}-02$ & $6.37 \mathrm{E}-03$ & $2.99 \mathrm{E}-03$ & $1.45 \mathrm{E}-03$ & $7.12 \mathrm{E}-04$ & $3.53 \mathrm{E}-04$ \\
$1.0 \mathrm{E}-09$ & $3.60 \mathrm{E}-02$ & $1.45 \mathrm{E}-02$ & $6.37 \mathrm{E}-03$ & $2.99 \mathrm{E}-03$ & $1.45 \mathrm{E}-03$ & $7.12 \mathrm{E}-04$ & $3.53 \mathrm{E}-04$ \\
$1.0 \mathrm{E}-10$ & $3.60 \mathrm{E}-02$ & $1.45 \mathrm{E}-02$ & $6.37 \mathrm{E}-03$ & $2.99 \mathrm{E}-03$ & $1.45 \mathrm{E}-03$ & $7.12 \mathrm{E}-04$ & $3.53 \mathrm{E}-04$ \\
\hline$e_{n}$ & $3.60 \mathrm{E}-02$ & $1.45 \mathrm{E}-02$ & $6.37 \mathrm{E}-03$ & $2.99 \mathrm{E}-03$ & $1.45 \mathrm{E}-03$ & $7.12 \mathrm{E}-04$ & $3.53 \mathrm{E}-04$ \\
\hline
\end{tabular}

TABle 2. Results for Example 5.2: Maximum errors via FOFDM (3.7) along with (3.8) after extrapolation.

\begin{tabular}{|c|ccccccc|}
\hline$\varepsilon$ & $n=16$ & $n=32$ & $n=64$ & $n=128$ & $n=256$ & $n=512$ & $n=1024$ \\
\hline $1.0 \mathrm{E}-04$ & $2.42 \mathrm{E}-02$ & $9.78 \mathrm{E}-03$ & $4.28 \mathrm{E}-03$ & $2.00 \mathrm{E}-03$ & $9.67 \mathrm{E}-04$ & $4.75 \mathrm{E}-04$ & $2.36 \mathrm{E}-04$ \\
$1.0 \mathrm{E}-05$ & $2.42 \mathrm{E}-02$ & $9.78 \mathrm{E}-03$ & $4.28 \mathrm{E}-03$ & $2.00 \mathrm{E}-03$ & $9.67 \mathrm{E}-04$ & $4.75 \mathrm{E}-04$ & $2.36 \mathrm{E}-04$ \\
$1.0 \mathrm{E}-06$ & $2.42 \mathrm{E}-02$ & $9.78 \mathrm{E}-03$ & $4.28 \mathrm{E}-03$ & $2.00 \mathrm{E}-03$ & $9.67 \mathrm{E}-04$ & $4.75 \mathrm{E}-04$ & $2.36 \mathrm{E}-04$ \\
$1.0 \mathrm{E}-08$ & $2.42 \mathrm{E}-02$ & $9.78 \mathrm{E}-03$ & $4.28 \mathrm{E}-03$ & $2.00 \mathrm{E}-03$ & $9.67 \mathrm{E}-04$ & $4.75 \mathrm{E}-04$ & $2.36 \mathrm{E}-04$ \\
$1.0 \mathrm{E}-09$ & $2.42 \mathrm{E}-02$ & $9.78 \mathrm{E}-03$ & $4.28 \mathrm{E}-03$ & $2.00 \mathrm{E}-03$ & $9.67 \mathrm{E}-04$ & $4.75 \mathrm{E}-04$ & $2.36 \mathrm{E}-04$ \\
$1.0 \mathrm{E}-10$ & $2.42 \mathrm{E}-02$ & $9.78 \mathrm{E}-03$ & $4.28 \mathrm{E}-03$ & $2.00 \mathrm{E}-03$ & $9.67 \mathrm{E}-04$ & $4.75 \mathrm{E}-04$ & $2.36 \mathrm{E}-04$ \\
\hline$e_{n}^{\text {ext }}$ & $2.42 \mathrm{E}-02$ & $9.78 \mathrm{E}-03$ & $4.28 \mathrm{E}-03$ & $2.00 \mathrm{E}-03$ & $9.67 \mathrm{E}-04$ & $4.75 \mathrm{E}-04$ & $2.36 \mathrm{E}-04$ \\
\hline
\end{tabular}

TABLE 3. Results for Example 5.2: Rates of convergence via FOFDM (3.7) along with (3.8) before extrapolation $n_{k}=$ $16,32,64,128,256,512$.

\begin{tabular}{|c|cccccc|}
\hline$\varepsilon$ & $r_{1}$ & $r_{2}$ & $r_{3}$ & $r_{4}$ & $r_{5}$ & $r_{6}$ \\
\hline $1.0 \mathrm{E}-04$ & 1.32 & 1.18 & 1.09 & 1.05 & 1.02 & 1.01 \\
$1.0 \mathrm{E}-05$ & 1.32 & 1.18 & 1.09 & 1.05 & 1.02 & 1.01 \\
$1.0 \mathrm{E}-06$ & 1.32 & 1.18 & 1.09 & 1.05 & 1.02 & 1.01 \\
$1.0 \mathrm{E}-08$ & 1.32 & 1.18 & 1.09 & 1.05 & 1.02 & 1.01 \\
$1.0 \mathrm{E}-09$ & 1.32 & 1.18 & 1.09 & 1.05 & 1.02 & 1.01 \\
$1.0 \mathrm{E}-10$ & 1.32 & 1.18 & 1.09 & 1.05 & 1.02 & 1.01 \\
\hline$r_{n}$ & 1.32 & 1.18 & 1.09 & 1.05 & 1.02 & 1.01 \\
\hline
\end{tabular}

Example 5.2. Consider problem (1.1)-(1.2) with

$$
\begin{gathered}
a(x)=-2 x^{3}, \quad b(x)=\exp \left(x^{2}\right), \\
f(x)=\left[2\left(1+\frac{2 x^{2}\left(1-x^{2}\right)}{\varepsilon}\right)-\exp \left(x^{2}\right)\right] \exp \left[\frac{x^{2}-1}{\varepsilon}\right] .
\end{gathered}
$$

Its exact solution is given by

$$
u(x)=\exp \left[-\frac{(1-x)(1+x)}{\varepsilon}\right] .
$$

The solution has a turning point at $x=0$. 
TABlE 4. Results for Example 5.2: Rates of convergence via FOFDM (3.7) along with (3.8) after extrapolation $n_{k}=$ $16,32,64,128,256,512$.

\begin{tabular}{|c|cccccc|}
\hline$\varepsilon$ & $r_{1}$ & $r_{2}$ & $r_{3}$ & $r_{4}$ & $r_{5}$ & $r_{6}$ \\
\hline $1.0 \mathrm{E}-04$ & 1.31 & 1.19 & 1.10 & 1.05 & 1.02 & 1.01 \\
$1.0 \mathrm{E}-05$ & 1.31 & 1.19 & 1.10 & 1.05 & 1.02 & 1.01 \\
$1.0 \mathrm{E}-06$ & 1.31 & 1.19 & 1.10 & 1.05 & 1.02 & 1.01 \\
$1.0 \mathrm{E}-08$ & 1.31 & 1.19 & 1.10 & 1.05 & 1.02 & 1.01 \\
$1.0 \mathrm{E}-09$ & 1.31 & 1.19 & 1.10 & 1.05 & 1.02 & 1.01 \\
$1.0 \mathrm{E}-10$ & 1.31 & 1.19 & 1.10 & 1.05 & 1.02 & 1.01 \\
\hline$r_{n}^{\text {ext }}$ & 1.31 & 1.19 & 1.10 & 1.05 & 1.02 & 1.01 \\
\hline
\end{tabular}

TABle 5. Results for Example 5.1: Maximum errors via FMFDM (4.21) along with (4.20) before extrapolation.

\begin{tabular}{|c|ccccccc|}
\hline$\varepsilon$ & $n=16$ & $n=32$ & $n=64$ & $n=128$ & $n=256$ & $n=512$ & $n=1024$ \\
\hline $1.0 \mathrm{E}-04$ & $1.43 \mathrm{E}-01$ & $9.44 \mathrm{E}-02$ & $6.04 \mathrm{E}-02$ & $3.77 \mathrm{E}-02$ & $2.29 \mathrm{E}-02$ & $1.36 \mathrm{E}-02$ & $7.84 \mathrm{E}-03$ \\
$1.0 \mathrm{E}-05$ & $1.43 \mathrm{E}-01$ & $9.44 \mathrm{E}-02$ & $6.04 \mathrm{E}-02$ & $3.77 \mathrm{E}-02$ & $2.29 \mathrm{E}-02$ & $1.36 \mathrm{E}-02$ & $7.84 \mathrm{E}-03$ \\
$1.0 \mathrm{E}-06$ & $1.43 \mathrm{E}-01$ & $9.44 \mathrm{E}-02$ & $6.04 \mathrm{E}-02$ & $3.77 \mathrm{E}-02$ & $2.29 \mathrm{E}-02$ & $1.36 \mathrm{E}-02$ & $7.84 \mathrm{E}-03$ \\
$1.0 \mathrm{E}-08$ & $1.43 \mathrm{E}-01$ & $9.44 \mathrm{E}-02$ & $6.04 \mathrm{E}-02$ & $3.77 \mathrm{E}-02$ & $2.29 \mathrm{E}-02$ & $1.36 \mathrm{E}-02$ & $7.84 \mathrm{E}-03$ \\
$1.0 \mathrm{E}-09$ & $1.43 \mathrm{E}-01$ & $9.44 \mathrm{E}-02$ & $6.04 \mathrm{E}-02$ & $3.77 \mathrm{E}-02$ & $2.29 \mathrm{E}-02$ & $1.36 \mathrm{E}-02$ & $7.84 \mathrm{E}-03$ \\
$1.0 \mathrm{E}-10$ & $1.43 \mathrm{E}-01$ & $9.44 \mathrm{E}-02$ & $6.04 \mathrm{E}-02$ & $3.77 \mathrm{E}-02$ & $2.29 \mathrm{E}-02$ & $1.36 \mathrm{E}-02$ & $7.84 \mathrm{E}-03$ \\
\hline$e_{n}$ & $1.43 \mathrm{E}-01$ & $9.44 \mathrm{E}-02$ & $6.04 \mathrm{E}-02$ & $3.77 \mathrm{E}-02$ & $2.29 \mathrm{E}-02$ & $1.36 \mathrm{E}-02$ & $7.84 \mathrm{E}-03$ \\
\hline
\end{tabular}

TABLE 6. Results for Example 5.1: Maximum errors via FMFDM (4.21) along with (4.20) after extrapolation.

\begin{tabular}{|c|ccccccc|}
\hline$\varepsilon$ & $n=16$ & $n=32$ & $n=64$ & $n=128$ & $n=256$ & $n=512$ & $n=1024$ \\
\hline $1.0 \mathrm{E}-04$ & $6.46 \mathrm{E}-02$ & $4.35 \mathrm{E}-02$ & $2.56 \mathrm{E}-02$ & $1.61 \mathrm{E}-02$ & $1.13 \mathrm{E}-02$ & $8.51 \mathrm{E}-03$ & $6.69 \mathrm{E}-03$ \\
$1.0 \mathrm{E}-05$ & $6.45 \mathrm{E}-02$ & $4.35 \mathrm{E}-02$ & $2.56 \mathrm{E}-02$ & $1.61 \mathrm{E}-02$ & $1.13 \mathrm{E}-02$ & $8.51 \mathrm{E}-03$ & $6.69 \mathrm{E}-03$ \\
$1.0 \mathrm{E}-06$ & $6.45 \mathrm{E}-02$ & $4.35 \mathrm{E}-02$ & $2.56 \mathrm{E}-02$ & $1.61 \mathrm{E}-02$ & $1.13 \mathrm{E}-02$ & $8.51 \mathrm{E}-03$ & $6.69 \mathrm{E}-03$ \\
$1.0 \mathrm{E}-08$ & $6.45 \mathrm{E}-02$ & $4.35 \mathrm{E}-02$ & $2.56 \mathrm{E}-02$ & $1.61 \mathrm{E}-02$ & $1.13 \mathrm{E}-02$ & $8.51 \mathrm{E}-03$ & $6.69 \mathrm{E}-03$ \\
$1.0 \mathrm{E}-09$ & $6.45 \mathrm{E}-02$ & $4.35 \mathrm{E}-02$ & $2.56 \mathrm{E}-02$ & $1.61 \mathrm{E}-02$ & $1.13 \mathrm{E}-02$ & $8.51 \mathrm{E}-03$ & $6.69 \mathrm{E}-03$ \\
$1.0 \mathrm{E}-10$ & $6.45 \mathrm{E}-02$ & $4.35 \mathrm{E}-02$ & $2.56 \mathrm{E}-02$ & $1.61 \mathrm{E}-02$ & $1.13 \mathrm{E}-02$ & $8.51 \mathrm{E}-03$ & $6.69 \mathrm{E}-03$ \\
\hline$e_{n}^{\text {ext }}$ & $6.45 \mathrm{E}-02$ & $4.35 \mathrm{E}-02$ & $2.56 \mathrm{E}-02$ & $1.61 \mathrm{E}-02$ & $1.13 \mathrm{E}-02$ & $8.51 \mathrm{E}-03$ & $6.69 \mathrm{E}-03$ \\
\hline
\end{tabular}

The maximum errors before extrapolation at all mesh points are evaluated using the fomulae

$$
e_{\varepsilon, n}:=\max _{0 \leq j \leq n}\left|u_{j}-U_{j}\right|
$$

for both FOFDM (3.7) along with (3.8) and FMFDM (4.21) along with (4.20). After extrapolation, the maximum errors are calculated as

$$
e_{\varepsilon, n}^{\text {ext }}:=\max _{0 \leq j \leq n}\left|u_{j}-U_{j}^{\text {ext }}\right| .
$$


TABLE 7. Results for Example 5.1: Rates of convergence via FMFDM (4.21) along with (4.20) before extrapolation $n_{k}=$ $16,32,64,128,256,512$.

\begin{tabular}{|c|cccccc|}
\hline$\varepsilon$ & $r_{1}$ & $r_{2}$ & $r_{3}$ & $r_{4}$ & $r_{5}$ & $r_{6}$ \\
\hline $1.0 \mathrm{E}-04$ & 0.60 & 0.64 & 0.68 & 0.72 & 0.76 & 0.79 \\
$1.0 \mathrm{E}-05$ & 0.60 & 0.64 & 0.68 & 0.72 & 0.76 & 0.79 \\
$1.0 \mathrm{E}-06$ & 0.60 & 0.64 & 0.68 & 0.72 & 0.76 & 0.79 \\
$1.0 \mathrm{E}-08$ & 0.60 & 0.64 & 0.68 & 0.72 & 0.76 & 0.79 \\
$1.0 \mathrm{E}-09$ & 0.60 & 0.64 & 0.68 & 0.72 & 0.76 & 0.79 \\
$1.0 \mathrm{E}-10$ & 0.60 & 0.64 & 0.68 & 0.72 & 0.76 & 0.79 \\
\hline$r_{n}$ & 0.60 & 0.64 & 0.68 & 0.72 & 0.76 & 0.79 \\
\hline
\end{tabular}

TABLE 8. Results for Example 5.1: Rates of convergence via FMFDM (4.21) along with (4.20) after extrapolation $n_{k}=$ 16, 32, 64, 128, 256, 512 .

\begin{tabular}{|c|cccccc|}
\hline$\varepsilon$ & $r_{1}$ & $r_{2}$ & $r_{3}$ & $r_{4}$ & $r_{5}$ & $r_{6}$ \\
\hline $1.0 \mathrm{E}-04$ & 0.57 & 0.77 & 0.67 & 0.51 & 0.41 & 0.35 \\
$1.0 \mathrm{E}-05$ & 0.57 & 0.77 & 0.67 & 0.51 & 0.41 & 0.35 \\
$1.0 \mathrm{E}-06$ & 0.57 & 0.77 & 0.67 & 0.51 & 0.41 & 0.35 \\
$1.0 \mathrm{E}-08$ & 0.57 & 0.77 & 0.67 & 0.51 & 0.41 & 0.35 \\
$1.0 \mathrm{E}-09$ & 0.57 & 0.77 & 0.67 & 0.51 & 0.41 & 0.35 \\
$1.0 \mathrm{E}-10$ & 0.57 & 0.77 & 0.67 & 0.51 & 0.41 & 0.35 \\
\hline$r_{n}^{\text {ext }}$ & 0.57 & 0.77 & 0.67 & 0.50 & 0.41 & 0.35 \\
\hline
\end{tabular}

TABLE 9. Results for Example 5.2: Maximum errors via FMFDM (4.21) along with (4.20) before extrapolation.

\begin{tabular}{|c|ccccccc|}
\hline$\varepsilon$ & $n=16$ & $n=32$ & $n=64$ & $n=128$ & $n=256$ & $n=512$ & $n=1024$ \\
\hline $1.0 \mathrm{E}-04$ & $9.85 \mathrm{E}-02$ & $8.58 \mathrm{E}-02$ & $5.88 \mathrm{E}-02$ & $3.75 \mathrm{E}-02$ & $2.29 \mathrm{E}-02$ & $1.36 \mathrm{E}-02$ & $7.84 \mathrm{E}-03$ \\
$1.0 \mathrm{E}-05$ & $9.85 \mathrm{E}-02$ & $8.58 \mathrm{E}-02$ & $5.88 \mathrm{E}-02$ & $3.75 \mathrm{E}-02$ & $2.29 \mathrm{E}-02$ & $1.36 \mathrm{E}-02$ & $7.84 \mathrm{E}-03$ \\
$1.0 \mathrm{E}-06$ & $9.85 \mathrm{E}-02$ & $8.58 \mathrm{E}-02$ & $5.88 \mathrm{E}-02$ & $3.75 \mathrm{E}-02$ & $2.29 \mathrm{E}-02$ & $1.36 \mathrm{E}-02$ & $7.84 \mathrm{E}-03$ \\
$1.0 \mathrm{E}-08$ & $9.85 \mathrm{E}-02$ & $8.58 \mathrm{E}-02$ & $5.88 \mathrm{E}-02$ & $3.75 \mathrm{E}-02$ & $2.29 \mathrm{E}-02$ & $1.36 \mathrm{E}-02$ & $7.84 \mathrm{E}-03$ \\
$1.0 \mathrm{E}-09$ & $9.85 \mathrm{E}-02$ & $8.58 \mathrm{E}-02$ & $5.88 \mathrm{E}-02$ & $3.75 \mathrm{E}-02$ & $2.29 \mathrm{E}-02$ & $1.36 \mathrm{E}-02$ & $7.84 \mathrm{E}-03$ \\
$1.0 \mathrm{E}-10$ & $9.85 \mathrm{E}-02$ & $8.58 \mathrm{E}-02$ & $5.88 \mathrm{E}-02$ & $3.75 \mathrm{E}-02$ & $2.29 \mathrm{E}-02$ & $1.36 \mathrm{E}-02$ & $7.83 \mathrm{E}-03$ \\
\hline$e_{n}$ & $9.85 \mathrm{E}-02$ & $8.58 \mathrm{E}-02$ & $5.88 \mathrm{E}-02$ & $3.74 \mathrm{E}-02$ & $2.29 \mathrm{E}-02$ & $1.35 \mathrm{E}-02$ & $7.83 \mathrm{E}-03$ \\
\hline
\end{tabular}

The numerical rates of convergence are computed by using the formula [8]:

$$
r_{k} \equiv r_{\varepsilon, k}:=\log _{2}\left(\widetilde{e}_{n_{k}} / \widetilde{e}_{2 n_{k}}\right), k=1,2, \ldots,
$$

where $\widetilde{e}$ stands for $e_{\varepsilon, n}$, and $e_{\varepsilon, n}^{\text {ext }}$, respectively.

Furthermore, we compute

$$
e_{n}=\max _{0 \leq \varepsilon \ll 1} e_{\varepsilon, n} \text { and } e_{n}^{\mathrm{ext}}=\max _{0 \leq \varepsilon \ll 1} e_{\varepsilon, n}^{\mathrm{ext}}
$$


TABle 10. Results for Example 5.2: Maximum errors via FMFDM (4.21) along with (4.20) after extrapolation.

\begin{tabular}{|c|ccccccc|}
\hline$\varepsilon$ & $n=16$ & $n=32$ & $n=64$ & $n=128$ & $n=256$ & $n=512$ & $n=1024$ \\
\hline $1.0 \mathrm{E}-04$ & $3.83 \mathrm{E}-02$ & $3.53 \mathrm{E}-02$ & $2.40 \mathrm{E}-02$ & $1.58 \mathrm{E}-02$ & $1.13 \mathrm{E}-02$ & $8.50 \mathrm{E}-03$ & $6.69 \mathrm{E}-03$ \\
$1.0 \mathrm{E}-05$ & $3.83 \mathrm{E}-02$ & $3.53 \mathrm{E}-02$ & $2.40 \mathrm{E}-02$ & $1.58 \mathrm{E}-02$ & $1.13 \mathrm{E}-02$ & $8.50 \mathrm{E}-03$ & $6.69 \mathrm{E}-03$ \\
$1.0 \mathrm{E}-06$ & $3.83 \mathrm{E}-02$ & $3.53 \mathrm{E}-02$ & $2.40 \mathrm{E}-02$ & $1.58 \mathrm{E}-02$ & $1.13 \mathrm{E}-02$ & $8.50 \mathrm{E}-03$ & $6.69 \mathrm{E}-03$ \\
$1.0 \mathrm{E}-08$ & $3.83 \mathrm{E}-02$ & $3.53 \mathrm{E}-02$ & $2.40 \mathrm{E}-02$ & $1.58 \mathrm{E}-02$ & $1.13 \mathrm{E}-02$ & $8.50 \mathrm{E}-03$ & $6.69 \mathrm{E}-03$ \\
$1.0 \mathrm{E}-09$ & $3.83 \mathrm{E}-02$ & $3.53 \mathrm{E}-02$ & $2.40 \mathrm{E}-02$ & $1.58 \mathrm{E}-02$ & $1.13 \mathrm{E}-02$ & $8.50 \mathrm{E}-03$ & $6.69 \mathrm{E}-03$ \\
$1.0 \mathrm{E}-10$ & $3.83 \mathrm{E}-02$ & $3.53 \mathrm{E}-02$ & $2.40 \mathrm{E}-02$ & $1.59 \mathrm{E}-02$ & $1.13 \mathrm{E}-02$ & $8.54 \mathrm{E}-03$ & $6.69 \mathrm{E}-03$ \\
\hline$e_{n}^{\text {ext }}$ & $3.83 \mathrm{E}-02$ & $3.53 \mathrm{E}-02$ & $2.40 \mathrm{E}-02$ & $1.59 \mathrm{E}-02$ & $1.13 \mathrm{E}-02$ & $8.54 \mathrm{E}-03$ & $6.69 \mathrm{E}-03$ \\
\hline
\end{tabular}

TABLE 11. Results for Example 5.2: Rates of convergence via FMFDM (4.21) along with (4.20) before extrapolation $n_{k}=16,32,64,128,256,512$.

\begin{tabular}{|c|cccccc|}
\hline$\varepsilon$ & $r_{1}$ & $r_{2}$ & $r_{3}$ & $r_{4}$ & $r_{5}$ & $r_{6}$ \\
\hline $1.0 \mathrm{E}-04$ & 0.27 & 0.54 & 0.63 & 0.75 & 0.97 & 0.98 \\
$1.0 \mathrm{E}-05$ & 0.24 & 0.55 & 0.64 & 0.70 & 0.75 & 0.79 \\
$1.0 \mathrm{E}-06$ & 0.22 & 0.54 & 0.65 & 0.71 & 0.75 & 0.79 \\
$1.0 \mathrm{E}-08$ & 0.20 & 0.54 & 0.65 & 0.71 & 0.76 & 0.79 \\
$1.0 \mathrm{E}-09$ & 0.20 & 0.54 & 0.65 & 0.71 & 0.76 & 0.79 \\
$1.0 \mathrm{E}-10$ & 0.20 & 0.54 & 0.65 & 0.71 & 0.76 & 0.79 \\
\hline$r_{n}$ & 0.20 & 0.54 & 0.65 & 0.71 & 0.76 & 0.79 \\
\hline
\end{tabular}

TABLE 12. Results for Example 5.2: Rates of convergence via FMFDM (4.21) along with (4.20) after extrapolation $n_{k}=$ $16,32,64,128,256,512$.

\begin{tabular}{|c|cccccc|}
\hline$\varepsilon$ & $r_{1}$ & $r_{2}$ & $r_{3}$ & $r_{4}$ & $r_{5}$ & $r_{6}$ \\
\hline $1.0 \mathrm{E}-04$ & 0.12 & 0.56 & 0.60 & 0.49 & 0.41 & 0.35 \\
$1.0 \mathrm{E}-05$ & 0.12 & 0.56 & 0.60 & 0.49 & 0.41 & 0.35 \\
$1.0 \mathrm{E}-06$ & 0.12 & 0.56 & 0.60 & 0.49 & 0.41 & 0.35 \\
$1.0 \mathrm{E}-08$ & 0.12 & 0.56 & 0.60 & 0.49 & 0.41 & 0.35 \\
$1.0 \mathrm{E}-09$ & 0.12 & 0.56 & 0.60 & 0.49 & 0.41 & 0.35 \\
$1.0 \mathrm{E}-10$ & 0.12 & 0.56 & 0.60 & 0.49 & 0.40 & 0.35 \\
\hline$r_{n}^{\text {ext }}$ & 0.12 & 0.56 & 0.60 & 0.49 & 0.40 & 0.35 \\
\hline
\end{tabular}

whereas the numerical rates of uniform convergence is computed as

$$
r_{n}:=\log _{2}\left(e_{n} / e_{2 n}\right) \text { and } r_{n}^{\text {ext }}:=\log _{2}\left(e_{n}^{\text {ext }} / e_{2 n}^{\text {ext }}\right) \text {. }
$$

\section{Discussion and concluding remarks}

With the view of checking the performance of some convergence acceleration techniques, in this paper, we designed a fitted mesh finite difference method (FMFDM) to solve a class of singularly perturbed turning-point problems whose solutions have boundary layers. The analysis shows that this method is 
robust with respect to the singular perturbation parameter $\varepsilon$ and is of order almost one. We also considered the fitted operator finite difference method (FOFDM) which we designed in [20]. This FOFDM is also robust with respect to $\varepsilon$. Then we investigated the effect of Richardson extrapolation on these methods and observed that, in both cases, this procedure improves the accuracy of the methods. However, the rates of convergence of these methods remain unimproved. This is in disagreement with a statement found in [27]. These conclusions drawn from our analysis are further confirmed by the numerical results found in tables 1-12. Moreover, comparing results in Table 1 to those in Table 9 we notice that the FOFDM is more accurate than the FMFDM.

Acknowledgment. Authors would like to acknowledgment anonyms referees for their valuable comments and suggestions which have improved the presentation of this work.

\section{References}

[1] L. R. Abrahamsson, A priori estimates for solutions of singular perturbations with a turning point, Stud. Appl. Math. 56 (1977), no. 1, 51-69.

[2] N. Adžić, Spectral approximation for single turning point problem, Z. Angew. Math. Mech. 72 (1992), no. 6, 621-624.

[3] _ Jacobi approximate solution of the boundary layer problem, Zb. Rad. Prirod.Mat. Fak. Ser. Math. 23 (1993), no. 1, 347-359.

[4] - Domain decomposition for spectral approximation of the layer solution, Zb. Rad. Prirod.-Mat. Fak. Ser. Math. 24 (1994), no. 1, 347-357.

[5] _ Jacobi polynomials in spectral approximation for shock layer problems, Zb. Rad. Prirod.-Mat. Fak. Ser. Math. 25 (1995), no. 1, 53-65.

[6] V. F. Andreev and A. M. Popov, Using Richardson's method to construct high-order accurate adaptive grids, Comput. Math. Model 10 (1999), no. 3, 227-238.

[7] A. E. Berger, H. Han, and R. B. Kellogg, A priori estimates and analysis of a numerical method for a turning point problem, Math. Comp. 42 (1984), no. 166, 465-492.

[8] E. P. Doolan, J. J. H. Miller, and W. H. A. Schilders, Uniform Numerical Methods for Problems with Initial and Boundary Layers, Boole Press, Dublin, 1980.

[9] R. Fößmeier, On Richardson extrapolation for finite difference methods on regular grids, Numer. Math. 55 (1989), no. 4, 451-462.

[10] A. Fröhner, T. Linß, and H.-G. Roos, Defect correction on Shishkin-type meshes, Numer. Algorithms. 26 (2001), no. 3, 281-299.

[11] A. Fröhner and H.-G. Roos, The $\varepsilon$-uniform convergence of a defect correction method on a Shishkin mesh, Appl. Numer. Math. 37 (2001), no. 1-2, 79-94.

[12] J. A. Gaunt, The deferred approach limit. II: interpenetrating lattices, Philos. Trans. R. Soc. Lond. Ser. A Math. Phys. Eng. Sci. 226 (1927), 350-361.

[13] D. C. Joyce, Survey of extrapolation process in numerical analysis, SIAM Rev. 13 (1971), no. $4,435-490$.

[14] P. A. Lagerstrom, Matched Asymptotic Expansions, Springer Verlag, New York, 1988.

[15] T. Linß, Robustness of an upwind finite difference scheme for semilinear convectiondiffusion problems with boundary turning points, J. Comput. Math. 21 (2003), no. 4, 401-410.

[16] T. Linß and R. Vulanović, Uniform methods for semilinear problems with an attractive turning point, Novi Sad J. Math. 31 (2001), no. 2, 99-114. 
[17] J. J. H. Miller, E. O'Riordan, and G. I. Shishkin, Fitted Numerical Methods for Singular Perturbation Problems, Word Scientific, Singapore, 1996.

[18] J. B. Munyakazi and K. C. Patidar, On Richardson extrapolation for fitted operator finite difference methods, Appl. Math. Comput. 201 (2008), no. 1-2, 465-480.

[19] L Limitations of Richardson's extrapolation for a high order fitted mesh method for self-adjoint singularly perturbed problems, J. Appl. Math. Comput. 32 (2010), no. $1,219-236$.

[20] — A fitted operator finite difference method for a singularly perturbed turning point problem whose solution has boundary layers, submitted.

[21] M. C. Natividad and M. Stynes, Richardson extrapolation for a convection-diffusion problem using a Shishkin mesh, Appl. Numer. Math. 45 (2003), no. 2-3, 315-329.

[22] K. C. Patidar, High order fitted operator numerical method for self-adjoint singular perturbation problems, Appl. Math. Comput. 171 (2005), no. 1, 547-566.

[23] _ High order parameter uniform numerical method for singular perturbation problems, Appl. Math. Comput. 188 (2007), no. 1, 720-733.

[24] L. F. Richardson, The deferred approach to the limit. I: Single lattice, Philos. Trans. R. Soc. Lond. Ser. A Math. Phys. Eng. Sci. 226 (1927), 299-349.

[25] H.-G. Roos and R. Vulanović, A higher order uniform convergence result for a turning point problem, Z. Anal. Anwend. 12 (1993), no. 4, 723-728.

[26] G. I. Shishkin, Robust novel high-order accurate numerical methods for singularly perturbed convection-diffusion problems, Math. Model. Anal. 10 (2005), no. 4, 393-412.

[27] G. I. Shishkin and L. P. Shishkina, The Richardson extrapolation technique for quasilinear parabolic singularly perturbed convection-diffusion equations, J. Phys.: Conf. Ser. 55 (2006), 203-213.

[28] R. Vulanović and P. A. Farrell, Continuous and numerical analysis of a multiple boundary turning point problem, SIAM J. Numer. Anal. 30 (1993), no. 5, 1400-1418.

Justin B. MunyaKaZi

Department of Mathematics and Applied Mathematics

University of the Western CAPe

Private Bag X17, Bellville 7535, South Africa

E-mail address: jmunyakazi@uwc.ac.za

Kailash C. Patidar

Department of Mathematics and Applied Mathematics

University OF THE Western CAPE

Private Bag X17, Bellville 7535, South Africa

E-mail address: kpatidar@uwc.ac.za 